\title{
Determination of Sleep State in Infants Using Respiratory Variability
}

\author{
GABRIEL G. HADDAD, HUAJIN J. JENG, TZE L. LAI, AND ROBERT B. MELLINS \\ Departments of Pediatrics (Pulmonary Division) and Mathematical Statistics, Columbia University, \\ New York, New York 10032
}

\begin{abstract}
Sleep staging has been conventionally performed using neurophysiologic and behavioral criteria. However, these criteria may not always be available. Since it is known that cardiorespiratory variables in rapid eye movement (REM) sleep are different from those in quiet sleep, we asked whether such variables can be used for the determination of sleep state. We studied nine normal fullterm infants at 1 and 4 months of life. Ventilation was measured using barometric plethysmography and the RR interval using a high accuracy $R$ wave detector. Electroencephalogram, electrooculogram, and postural muscle electromyogram were recorded using surface electrodes and behavioral criteria applied. Means of RR interval, respiratory cycle time and tidal volume, and coefficients of variation of the same variables, were obtained for 30-s intervals throughout each sleep study. The KolmogorovSmirnov distances between REM and quiet sleep were larger for the coefficients of variation than for the means at both ages for all variables. Moreover, coefficient of variation of respiratory cycle time was found to provide the largest separation between REM and quiet sleep. In view of this result, we developed a statistical decision rule using coefficient of variation of respiratory cycle time for the classification of REM and quiet sleep in blocks of 5-min periods. Each study was divided into 5-min epochs and this rule was applied to each epoch. Of 85 epochs staged as quiet sleep by neurophysiologic and behavioral criteria, 79 epochs (or 93\%) were classified correctly as quiet sleep using our decision rule. Of 85 epochs staged as REM sleep, 84 were classified as REM sleep and only one misclassified as quiet sleep. Five additional infants, whose results did not enter into the formulation of the decision rule, were used as a subsequent example to test the rule. The results of these five infants were similar to those of the previous nine others. We conclude that in young infants 1) the variability of cardiorespiratory parameters can separate quiet from nonquiet sleep better than the mean values and 2) sleep staging using a decision rule based on coefficient of variation of respiratory cycle time can be performed with high degree of accuracy. (Pediatr Res 21: 556-562, 1987)
\end{abstract}

\section{Abbreviations}

REM, rapid eye movement

EEG, electroencephalogram

KSD, Kolmogorov-Smirnov distance

Received August 27, 1986; accepted January 6, 1987

Address for correspondence Dr. Gabriel G. Haddad, Columbia University College of Physicians and Surgeons, 630 West 168th Street, New York, NY, 10032.

This research was supported by NIH Training Grant HL 07421, NIH Contract HD5-2856, and additional support from A. H. Robins Company Inc. This work was done during the tenure by G. G. H. of an Established Investigatorship from the American Heart Association. $v$ (Ttot), coefficient of variation of respiratory cycle time EOG, electrooculogram

EMG, electromyogram

$V t$, tidal volume

In the past 1-2 decades, monitoring heart rate and respiration in the fetus or in early postnatal life has increased substantially for clinical or research purposes in humans and animals (1-14). Furthermore, technical advances involving computerized techniques have made it possible not only to record or analyze cardiorespiratory variables but to do so over prolonged periods of time. For instance, studies during gestation, labor, or in early postnatal life have been performed over several hours or even days $(5-8,13)$.

One major concern in such studies is that during long periods of time, the state of consciousness of the subject can change. For example, the subject can change from, say, REM to quiet sleep, REM sleep to wakefulness, and so forth. Since the state of consciousness has long been recognized as an important factor that determines cardiorespiratory function $(1-4,6,8)$, studies performed in clinical or research laboratories take this into account using behavioral or neurophysiologic techniques or both $(8,15-17)$. However, cardiorespiratory studies on infants may also be done in a hospital setting (on the ward or the intensive care unit) or even at the home. One example of the latter was undertaken by Southall et al. (18) on more than 9000 infants. In these studies, neurophysiologic signals such as the EEG, the EOG, or the postural muscle EMG are usually not available. Moreover, behavioral coding of sleep state which requires the continuous presence of qualified personnel during these studies may not be possible. Hence, there is increased need to make measurements of state of consciousness without resorting to neurophysiologic signals.

It is known that cardiorespiratory function in REM sleep is different from that in quiet sleep $(1,3,6,8,9,19)$ and some efforts (20-22) have been made to distinguish states of consciousness in adult humans using heart rate. Such investigations have been scarce in the young, and the recent attempts of Harper et al. $(23,24)$ provide important initial observations in this direction. In the current work we study how, and with what confidence, one can use this difference in cardiorespiratory function to distinguish REM from quiet sleep in early life. In order to address this question, we examined the RR interval (inverse of heart rate), the respiratory cycle time or Ttot (inverse of respiratory frequency), and Vt in REM and quiet sleep in normal infants at 1 and 4 months of age. In these studies, neurophysiologic signals and direct behavioral observations were recorded. Based on our analysis of these studies, we propose a statistical decision rule using the respiratory frequency. We show that, when compared to staging based on neurophysiologic and be- 
havioral data, staging using this decision rule can attain a very high degree of accuracy.

\section{METHODS}

Infant subjects. Nine normal full-term infants were included and studied at 1 and 4 months of age (18 studies). A full description of the population has been given previously (25). In brief, all infants were full term and all pregnancies and deliveries were uncomplicated. Birth weights ranged between 2700 to 4040 g. All studies were performed after a midmorning feed during natural sleep. This sleep period averaged $2-3 \mathrm{~h}$ in our laboratory. During the latter half of this sleep period, infants were given $\mathrm{CO}_{2}$ to breathe as part of a different protocol; the data from these latter studies were not included in our current analysis. Each study was terminated by the natural awakening of the infant, a state characterized by low-voltage, high-frequency EEG, increased bodily movements, and open eyes. No infant was on any medication. In every study, the same techniques and the same laboratory environment were used and the same personnel performed the studies and handled infants. A written informed consent was obtained from the parents of each infant.

In addition to these nine infants studied at 1 and 4 months of age, we also conducted similar studies on five other infants at 1 or 4 months of age. The results of these additional studies were used to test the performance of the statistical decision rule that was based on the studies of the nine infants.

Sleep staging. Standard skin surface electrodes were used to record three neurophysiologic signals: a C4-A1 EEG, a bipolar EOG, and a bipolar submental EMG $(17,25)$. The resistance of these electrodes was less that $10 \mathrm{kohms}$, and the signals were noise free. A behavioral code $(15,16)$ that described the infant's somatic activities during sleep was recorded throughout each study by an observer. Using the EEG, EOG, EMG, and behavioral criteria, sleep was staged visually on 30-s epochs by two independent observers. In 85 to $90 \%$ of the instances, both observers agreed on the sleep state. In the remaining periods, staging was performed after careful rereview of the sleep records $(5,17)$. Sleep staging observers did not take into consideration changes in the respiratory signal.

$R R$ interval measurement. Electrocardiogram surface electrodes were positioned on the chest anteriorly as described previously (5) to obtain a unidirectional polygraph recording with a single prominent $\mathrm{R}$ wave which had a sharp peak. Each $\mathrm{RR}$ interval of this wide-band electrocardiogram signal $(0.5$ to 2000 $\mathrm{Hz}$ ) was measured with an accuracy of $0.2 \mathrm{~ms}$ using a digital preprocessor designed in this laboratory $(26)$. The data were carefully reviewed and edited and less than $0.1 \%$ of the 10,000 to $15,000 \mathrm{RR}$ intervals of each study were rejected as artifact.

Respiratory measurements. To avoid any sensory stimulation to breathing, ventilation was recorded wholly noninvasively using barometric plethysmography. The description of this method and details of its implementation have been described in detail elsewhere $(25,27)$. This method relies on the difference between the volume of air inspired and the volume of chest expansion. This difference, which results from heating and humidification of inspired air, produces an increase in pressure inside the chamber during inspiration; a decrease in pressure inside the box occurs during expiration. The temperature and humidity inside the box, the concentration of $\mathrm{CO}_{2}$ and $\mathrm{O}_{2}$, as well as the skin temperature of the infant, were recorded continuously during each study. The temperature of the chamber was approximately $25^{\circ} \mathrm{C}$ and the relative humidity was kept approximately between 40 and $60 \%$. We digitized the respiratory waveform at $25 \mathrm{~Hz}$ with the aid of a minicomputer and measured Vt and Ttot. All artifact-free breaths were included in the analysis and this amounted to about $2000-3000$ breaths per study.

Statistical and analytical techniques. To compare the distribution of a cardiorespiratory variable during quiet sleep with that during REM sleep, we plotted the relative frequency poly- gons (28) of the variable during quiet sleep and during REM sleep of each study. The KSD between the distribution function $\mathrm{Q}$ during quiet sleep and the distribution function $\mathrm{R}$ during REM sleep was computed for each study. We used the KSD $(=\max |\mathrm{Q}(\mathrm{x})-\mathrm{R}(\mathrm{x})|)$ to perform the Kolmogorov-Smirnov twosample test for statistically significant differences between $Q$ and $R$. The value $x=x_{0}$ that maximizes $|Q(x)-R(x)|$ is called the Kolmogorov-Smirnov cut (29) and we used it for nonparametric classification of REM and quiet sleep on the basis of the cardiorespiratory variable under consideration. As shown by Stoller (30), the rule that minimizes the sum of misclassification probabilities classifies the sleep state according to whether the observed variable is above or below the threshold value $\mathrm{x}_{0}[$ i.e. in the case $\mathrm{Q}\left(\mathrm{x}_{0}\right) \geq \mathrm{R}\left(\mathrm{x}_{0}\right)$, the state is classified as quiet sleep if $\mathrm{x} \leq$ $x_{0}$ and REM sleep if $x>x_{0}$. Moreover, for this rule, the sum of misclassification probabilities is equal to $1-\operatorname{KSD}(30)$.

Our analysis was performed on epochs of two different sizes. The first is a $30-\mathrm{s}$ epoch and the second is a 5-min epoch. The rationale for the use of the first is that generally electroencephalographers use this time frame for the examination of the EEG and other neurophysiologic and behavioral criteria. The rationale for the use of the longer period stems from the fact that investigators also take into consideration the state of the infant in several preceding and subsequent epochs while staging a current epoch. Therefore, in practice, sleep staging is performed on the basis of data spanning several successive minutes.

\section{RESULTS}

Sleep state and cardiorespiratory variables. Figure 1 shows the respiratory waveform (top) and ECG (bottom) in REM sleep
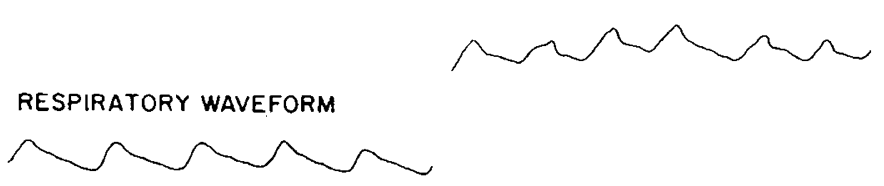

EOG

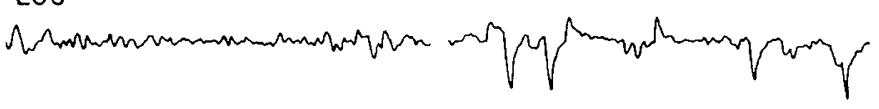

EEG

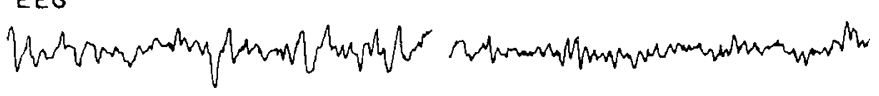
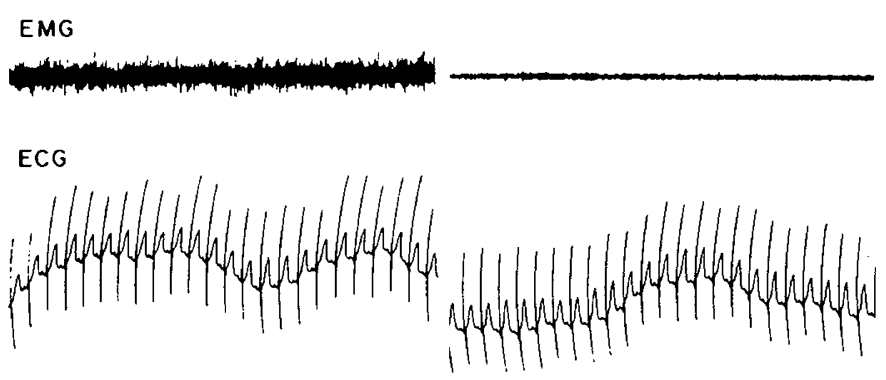

Fig. 1. Polygraphic recordings of the respiratory waveform (top) and ECG (bottom) in one infant at 4 months of age. Neurophysiologic signals (EOG, EEG, and EMG) are used to stage sleep. Note the high voltage/ low frequency EEG, the absence of rapid eye-movement swings on the EOG trace, and the increased muscle tone on the EMG trace in quiet sleep (left). In contrast, note the REM on the EOG tracing, the low voltage/high frequency EEG, and the low muscle tone in REM sleep (right). Each panel represents approximately $10 \mathrm{~s}$ of data in quiet or REM sleep. 
(left) and quiet sleep (right) in one infant at 4 months of age. The sleep staging was based on three neurophysiologic signals, i.e. EOG, EEG and postural muscle (chin) EMG (Fig. 1), and behavioral criteria. Although there is little apparent difference in the RR interval on the ECG waveform between REM and quiet sleep, Figure 1 shows a difference in the breathing pattern between these two sleep states.

In order to compare the respiratory pattern and the RR interval between REM and quiet sleep for all infants at both ages, we computed the means and coefficients of variation for RR interval, Ttot, and Vt over successive 30 -s periods for each study. These means and coefficients of variation will be denoted by $\mathrm{m}(\mathrm{RR}), \mathrm{m}(\mathrm{Ttot}), \mathrm{m}(\mathrm{Vt}), \mathrm{v}(\mathrm{RR}), \mathrm{v}(\mathrm{Ttot})$, and $\mathrm{v}(\mathrm{Vt})$, respectively, and Figure 2 shows their relative frequency polygons in REM and quiet sleep in the same study as shown in Figure 1. Figure 2 demonstrates that the difference between the relative frequency polygons of quiet and REM sleep is biggest for $v$ (Ttot) and is minimal for $m(R R)$ and $m$ (Ttot). Note that the frequency distribution of $v$ (Ttot) for quiet sleep is concentrated at low values of $v($ Ttot $)$; in fact $70 \%$ of the values are less than 0.12 . On the other hand, the frequency distribution of $v$ (Ttot) in REM sleep shows much greater dispersion and only $3 \%$ of the values are below the same threshold. This difference between REM and quiet sleep is shown more clearly in Figure 3 which plots the cumulative distribution functions of $v(T$ tot $)$ for REM and quiet sleep. The KSD between the distribution functions in Figure 3 is 0.66 and the Kolmogorov-Smirnov cut (see "Methods") occurs at a v(Ttot) $=0.11$.

Tables 1 and 2 present the KSD and Kolmogorov-Smirnov cuts for the $m(R R), m(T$ tot) and $m(V t)$ (Table 1) and $v(R R)$, $\mathrm{v}(\mathrm{Ttot}$ ) and $\mathrm{v}(\mathrm{Vt})$ (Table 2) in all infants and at both 1 and 4 months of age. Note that the KSDs in Table 1 are lower than those in Table 2 and that the KSDs for $v($ Ttot) in Table 2 are generally higher than those for $v(V t)$ and $v(R R)$. All KSDs for $v($ Ttot) at both 1 and 4 months of age exceed the critical values of the Kolmogorov-Smirnov test at the 0.01 level, showing highly significant differences in Ttot variability between REM and quiet sleep in each infant at both ages. In contrast, although the KSD

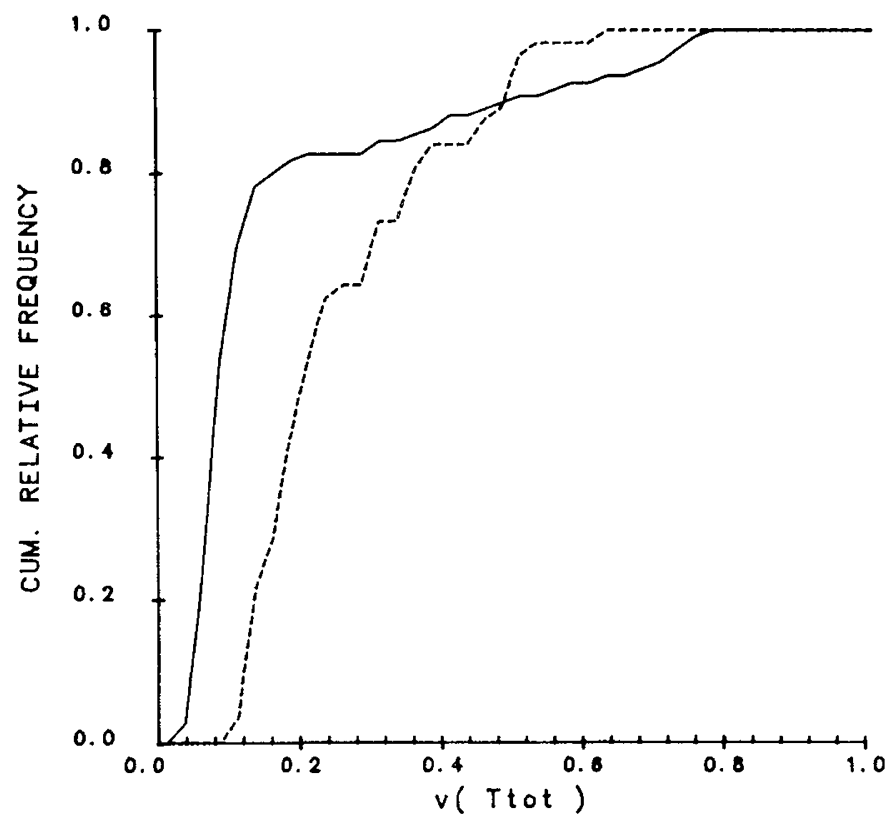

Fig. 3. Cumulative distribution functions for $v(T$ tot) in the same infant as in Figures 1 and 2 in quiet sleep (solid line) and REM sleep (dashed line). Note that the cumulative relative frequency in quiet sleep reaches about $70 \%$ at a v(Ttot) value of 0.12 ; in contrast, only $3 \%$ of $v($ Ttot $)$ values in REM sleep are below the same threshold.
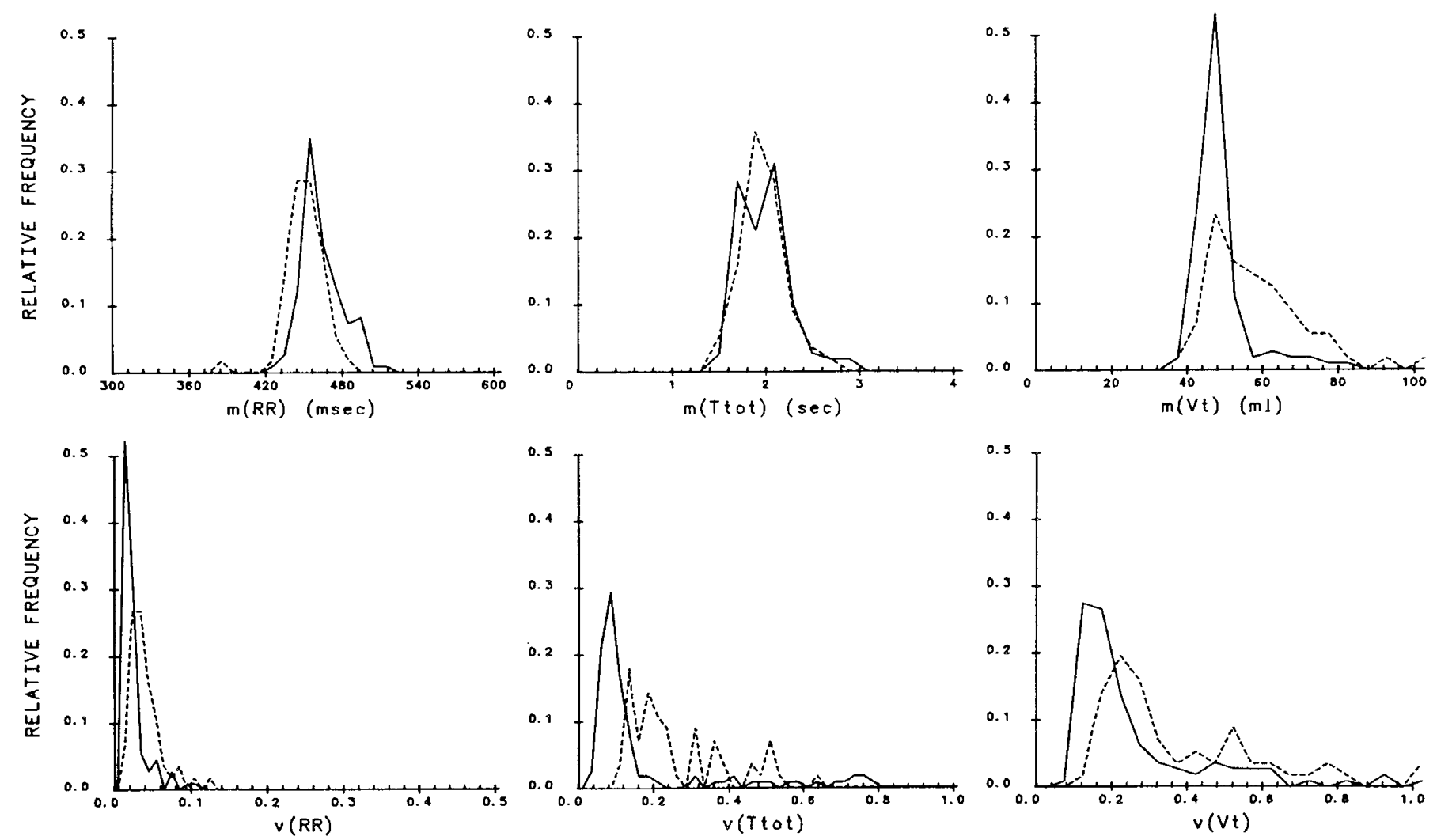

Fig. 2. This figure shows six relative frequency polygons of the means of RR interval $[m(R R)]$, total respiratory cycle time $[m(T t o t)]$, and $V t$ $[m(V t)]$, and coefficients of variation for the same variables in the same infant as in Figure 1. Each polygon represents the distribution of the means of coefficients of variation of the variable under consideration for the entire REM or quiet sleep period. Each mean or coefficient of variation is computed from a 30-s period. The solid line polygon represents quiet sleep and the dashed line polygon represents REM sleep. Note that the difference between the polygons is biggest for $v($ Ttot). 
Table 1. KSD and cuts (between parentheses) of means of RR interval $[m(R R)]$, respiratory cycle time or Ttot [m(Ttot)], and tidal volume $[\mathrm{m}(\mathrm{Vt})]$ obtained from 30-s periods in REM and quiet sleep in nine infants at 1 and 4 mo of age

\begin{tabular}{|c|c|c|c|c|c|c|}
\hline \multirow[b]{2}{*}{ Baby } & \multicolumn{2}{|c|}{$\mathrm{m}(\mathrm{RR})$} & \multicolumn{2}{|c|}{$\mathrm{m}($ Ttot $)$} & \multicolumn{2}{|c|}{$\mathrm{m}(\mathrm{Vt})$} \\
\hline & $1 \mathrm{mo}$ & $4 \mathrm{mo}$ & $1 \mathrm{mo}$ & $4 \mathrm{mo}$ & $1 \mathrm{mo}$ & $4 \mathrm{mo}$ \\
\hline 1 & $\begin{array}{l}0.23 \\
(0.385)\end{array}$ & $\begin{array}{c}0.31^{*} \\
(0.445)\end{array}$ & $\begin{array}{l}0.54^{*} \\
(1.5)\end{array}$ & $\begin{array}{l}0.098 \\
(1.7)\end{array}$ & $\begin{array}{c}0.70^{*} \\
(27.5)\end{array}$ & $\begin{array}{c}0.47^{*} \\
(47.5)\end{array}$ \\
\hline 2 & $\begin{array}{c}0.53^{*} \\
(0.455)\end{array}$ & $\begin{array}{c}0.31 \\
(0.495)\end{array}$ & $\begin{array}{l}0.36^{*} \\
(1.9)\end{array}$ & $\begin{array}{l}0.37^{*} \\
(2.3)\end{array}$ & $\begin{array}{c}0.59^{*} \\
(27.5)\end{array}$ & $\begin{array}{c}0.70^{*} \\
(67.5)\end{array}$ \\
\hline 3 & $\begin{array}{c}0.63^{*} \\
(0.415)\end{array}$ & $\begin{array}{c}0.46^{*} \\
(0.495)\end{array}$ & $\begin{array}{l}0.54^{*} \\
(1.1)\end{array}$ & $\begin{array}{c}0.24 \\
(1.7)\end{array}$ & $\begin{array}{r}0.22 \\
(12.5)\end{array}$ & $\begin{array}{r}0.04 \\
(42.5)\end{array}$ \\
\hline 4 & $\begin{array}{l}0.21 \\
(0.425)\end{array}$ & $\begin{array}{c}0.62^{*} \\
(0.455)\end{array}$ & $\begin{array}{l}0.70^{*} \\
(1.3)\end{array}$ & $\begin{array}{l}0.55^{*} \\
(1.5)\end{array}$ & $\begin{array}{c}0.64^{*} \\
(17.5)\end{array}$ & $\begin{array}{r}0.24 \\
(22.5)\end{array}$ \\
\hline 5 & $\begin{array}{c}0.46^{*} \\
(0.525)\end{array}$ & $\begin{array}{c}0.21 \\
(0.495)\end{array}$ & $\begin{array}{l}0.58^{*} \\
(1.3)\end{array}$ & $\begin{array}{l}0.83^{*} \\
(1.5)\end{array}$ & $\begin{array}{r}0.26 \\
(17.5)\end{array}$ & $\begin{array}{r}0.12 \\
(57.5)\end{array}$ \\
\hline 6 & $\begin{array}{c}0.45^{*} \\
(0.445)\end{array}$ & $\begin{array}{c}0.47^{*} \\
(0.465)\end{array}$ & $\begin{array}{c}0.25 \\
(1.9)\end{array}$ & $\begin{array}{c}0.28 \\
(2.1)\end{array}$ & $\begin{array}{c}0.73^{*} \\
(22.5)\end{array}$ & $\begin{array}{r}0.19 \\
(47.5)\end{array}$ \\
\hline 7 & $\begin{array}{c}0.46^{*} \\
(0.425)\end{array}$ & $\begin{array}{c}0.24 \\
(0.455)\end{array}$ & $\begin{array}{c}0.22 \\
(2.1)\end{array}$ & $\begin{array}{c}0.28 \\
(1.7)\end{array}$ & $\begin{array}{c}0.48^{*} \\
(22.5)\end{array}$ & $\begin{array}{c}0.34^{*} \\
(22.5)\end{array}$ \\
\hline 8 & $\begin{array}{c}0.90^{*} \\
(0.575)\end{array}$ & $\begin{array}{c}0.37^{*} \\
(0.455)\end{array}$ & $\begin{array}{l}0.53^{*} \\
(2.1)\end{array}$ & $\begin{array}{l}0.66^{*} \\
(1.9)\end{array}$ & $\begin{array}{r}0.24 \\
(27.5)\end{array}$ & $\begin{array}{c}0.35^{*} \\
(72.5)\end{array}$ \\
\hline 9 & $\begin{array}{c}0.44^{*} \\
(0.475)\end{array}$ & $\begin{array}{c}0.47^{*} \\
(0.505)\end{array}$ & $\begin{array}{c}0.13 \\
(1.9)\end{array}$ & $\begin{array}{l}0.39^{*} \\
(1.9)\end{array}$ & $\begin{array}{c}0.59^{*} \\
(27.5)\end{array}$ & $\begin{array}{c}0.29^{*} \\
(42.5)\end{array}$ \\
\hline Mean $\pm \mathrm{SD}$ of $\mathrm{KSD}$ & $0.48 \pm 0.21$ & $0.38 \pm 0.13$ & $0.43 \pm 0.19$ & $0.41 \pm 0.23$ & $0.49 \pm 0.20$ & $0.30 \pm 0.2$ \\
\hline
\end{tabular}

${ }^{*} p<0.05$, Kolmogorov-Smirnov test for significance.

Table 2. KSD and cuts (between parentheses) of coefficients of variations of $R R$ interval $[v(R R)]$, respiratory cycle time or Ttot $[\mathrm{v}($ Ttot $)]$ and tidal volume $[\mathrm{v}(\mathrm{Vt})]$ obtained from 30-s periods in REM and quiet sleep in nine infants at 1 and $4 \mathrm{mo}$ of age

\begin{tabular}{|c|c|c|c|c|c|c|}
\hline \multirow[b]{2}{*}{ Baby } & \multicolumn{2}{|c|}{$v(R R)$} & \multicolumn{2}{|c|}{$v($ Ttot $)$} & \multicolumn{2}{|c|}{$v(V t)$} \\
\hline & $1 \mathrm{mo}$ & $4 \mathrm{mo}$ & $1 \mathrm{mo}$ & $4 \mathrm{mo}$ & $1 \mathrm{mo}$ & $4 \mathrm{mo}$ \\
\hline 1 & $\begin{array}{c}0.76^{*} \\
(0.015)\end{array}$ & $\begin{array}{c}0.49^{*} \\
(0.025)\end{array}$ & $\begin{array}{c}0.74^{*} \\
(0.138)\end{array}$ & $\begin{array}{c}0.66^{*} \\
(0.113)\end{array}$ & $\begin{array}{c}0.50^{*} \\
(0.225)\end{array}$ & $\begin{array}{c}0.39^{*} \\
(0.175)\end{array}$ \\
\hline 2 & $\begin{array}{c}0.79^{*} \\
(0.025)\end{array}$ & $\begin{array}{c}0.46^{*} \\
(0.035)\end{array}$ & $\begin{array}{c}0.85^{*} \\
(0.163)\end{array}$ & $\begin{array}{c}0.74^{*} \\
(0.088)\end{array}$ & $\begin{array}{c}0.67^{*} \\
(0.225)\end{array}$ & $\begin{array}{c}0.28 \\
(0.125)\end{array}$ \\
\hline 3 & $\begin{array}{c}0.62^{*} \\
(0.025)\end{array}$ & $\begin{array}{c}0.38^{*} \\
(0.065)\end{array}$ & $\begin{array}{c}0.55^{*} \\
(0.163)\end{array}$ & $\begin{array}{c}0.58^{*} \\
(0.088)\end{array}$ & $\begin{array}{c}0.14 \\
(0.225)\end{array}$ & $\begin{array}{c}0.41^{*} \\
(0.175)\end{array}$ \\
\hline 4 & $\begin{array}{c}0.77^{*} \\
(0.015)\end{array}$ & $\begin{array}{l}0.15 \\
(0.025)\end{array}$ & $\begin{array}{c}0.76^{*} \\
(0.163)\end{array}$ & $\begin{array}{c}0.41^{*} \\
(0.138)\end{array}$ & $\begin{array}{c}0.79^{*} \\
(0.225)\end{array}$ & $\begin{array}{c}0.28 \\
(0.125)\end{array}$ \\
\hline 5 & $\begin{array}{c}0.67^{*} \\
(0.035)\end{array}$ & $\begin{array}{c}0.39^{*} \\
(0.035)\end{array}$ & $\begin{array}{c}0.73^{*} \\
(0.188)\end{array}$ & $\begin{array}{c}0.83^{*} \\
(0.088)\end{array}$ & $\begin{array}{c}0.73^{*} \\
(0.275)\end{array}$ & $\begin{array}{c}0.64^{*} \\
(0.125)\end{array}$ \\
\hline 6 & $\begin{array}{l}0.92^{*} \\
(0.025)\end{array}$ & $\begin{array}{c}0.65^{*} \\
(0.025)\end{array}$ & $\begin{array}{c}0.86^{*} \\
(0.138)\end{array}$ & $\begin{array}{c}0.67^{*} \\
(0.138)\end{array}$ & $\begin{array}{c}0.77^{*} \\
(0.28)\end{array}$ & $\begin{array}{c}0.72^{*} \\
(0.225)\end{array}$ \\
\hline 7 & $\begin{array}{c}0.75^{*} \\
(0.015)\end{array}$ & $\begin{array}{c}0.70^{*} \\
(0.015)\end{array}$ & $\begin{array}{c}0.75^{*} \\
(0.138)\end{array}$ & $\begin{array}{c}0.72^{*} \\
(0.138)\end{array}$ & $\begin{array}{c}0.51^{*} \\
(0.175)\end{array}$ & $\begin{array}{c}0.66^{*} \\
(0.175)\end{array}$ \\
\hline 8 & $\begin{array}{c}0.91^{*} \\
(0.025)\end{array}$ & $\begin{array}{l}0.25 \\
(0.075)\end{array}$ & $\begin{array}{c}0.81^{*} \\
(0.113)\end{array}$ & $\begin{array}{c}0.54^{*} \\
(0.113)\end{array}$ & $\begin{array}{c}0.12 \\
(0.175)\end{array}$ & $\begin{array}{l}0.22 \\
(0.225)\end{array}$ \\
\hline 9 & $\begin{array}{c}0.55^{*} \\
(0.015)\end{array}$ & $\begin{array}{c}0.48^{*} \\
(0.025)\end{array}$ & $\begin{array}{c}0.76^{*} \\
(0.163)\end{array}$ & $\begin{array}{c}0.80^{*} \\
(0.138)\end{array}$ & $\begin{array}{c}0.56^{*} \\
(0.275)\end{array}$ & $\begin{array}{c}0.66^{*} \\
(0.225)\end{array}$ \\
\hline Mean \pm SD of KSD & $0.75 \pm 0.12$ & $0.44 \pm 0.17$ & $0.76 \pm 0.09$ & $0.66 \pm 0.13$ & $0.53 \pm 0.25$ & $0.47 \pm 0.2$ \\
\hline
\end{tabular}

${ }^{*} p<0.05$, Kolmogorov-Smirnov test for significance. 
for $v(R R)$ is as high as $v(T$ tot $)$ at 1 month of age, it does not even reach significance (at the 0.01 level) in two infants and it is substantially lower than $\mathrm{v}$ (Ttot) at 4 months of age. Note also that the Kolmogorov-Smirnov cuts for $\mathrm{v}(\mathrm{Ttot})$ are consistent from one infant to another and range between 0.08 and 0.19 for all infants at both ages.

Determination of sleep state using $v$ (Ttot). Thirty-S Epochs. Table 2 shows that $v$ (Ttot) has the largest KSD at both ages. As stated in the Methods section, the larger the KSD, the smaller is the sum of misclassification probabilities $(=1-\mathrm{KSD})$ when classification or discrimination is based on the Kolmogorov-Smirnov

Table 3. Correct classification probabilities of the rule that classifies a 30-s epoch as in REM (or quiet) sleep according to $v($ Ttot $)>(\leq) 0.15$ in sleeping infants at 1 and 4 mo of age (see

\begin{tabular}{|c|c|c|c|c|}
\hline \multirow[b]{2}{*}{ Baby } & \multicolumn{2}{|c|}{ 1-mo old } & \multicolumn{2}{|c|}{ 4-mo old } \\
\hline & Quiet & REM & Quiet & REM \\
\hline \multicolumn{5}{|c|}{ Studies on the initial 9 infants } \\
\hline 1 & 0.90 & 0.83 & 0.78 & 0.79 \\
\hline 2 & 0.87 & 0.95 & 0.96 & 0.48 \\
\hline 3 & 0.74 & 0.76 & 0.99 & 0.39 \\
\hline 4 & 0.85 & 0.88 & 0.70 & 0.71 \\
\hline 5 & 0.68 & 1.00 & 0.98 & 0.56 \\
\hline 6 & 0.87 & 0.99 & 0.83 & 0.84 \\
\hline 7 & 0.78 & 0.97 & 0.89 & 0.83 \\
\hline 8 & 0.89 & 0.82 & 0.83 & 0.68 \\
\hline 9 & 0.88 & 0.87 & 0.89 & 0.91 \\
\hline \multicolumn{5}{|c|}{ Studies on the 5 additional infants } \\
\hline \multicolumn{2}{|c|}{ aby (age) } & Quiet & \multicolumn{2}{|c|}{ REM } \\
\hline \multicolumn{2}{|c|}{$10(1 \mathrm{mo})$} & 0.91 & \multicolumn{2}{|r|}{0.67} \\
\hline \multicolumn{2}{|c|}{$11(1 \mathrm{mo})$} & 0.78 & \multicolumn{2}{|r|}{0.94} \\
\hline \multicolumn{2}{|c|}{$12(1 \mathrm{mo})$} & 0.93 & \multicolumn{2}{|r|}{0.88} \\
\hline \multicolumn{2}{|c|}{$13(1 \mathrm{mo})$} & 0.87 & \multicolumn{2}{|r|}{0.82} \\
\hline \multicolumn{2}{|c|}{$14(4 \mathrm{mo})$} & 0.96 & \multicolumn{2}{|r|}{0.55} \\
\hline
\end{tabular}

cut. Based on these results, therefore, $v(T$ tot $)$ is the best variable for discriminating between REM and quiet sleep among the 6 variables considered. Since the Kolmogorov-Smirnov cut for $v($ Ttot) varies within a narrow range $(0.08-0.19)$ in all infants at both ages, we use a value in the middle of the range, say 0.15 , as a common threshold for all infants at both ages to determine the sleep state of a given 30-second epoch on the basis of v(Ttot). Thus, we classify an epoch as REM sleep if the $\mathrm{v}$ (Ttot) value is above 0.15 and as quiet sleep if the value is below 0.15 . The performance of this classification rule is shown in Table 3 which reports the relative frequency of correctly classified 30 -s epochs in each study.

Five-Min Epochs. The classification rule above is based on data in a single 30 -s block assuming that sleep staging is performed on each of these blocks independently of previous or subsequent blocks. However, sleep staging based on neurophysiologic and behavioral criteria of a current block is generally done taking into account the tentative sleep state of several previous and subsequent blocks, spanning few successive minutes. Therefore, as explained in "Methods," we divided each study into longer blocks, each of 5-min duration, and considered classification rules based on the (10) values of $v$ (Ttot), which represents short-term $(30 \mathrm{~s})$ Ttot variability, during each 5 -min block. Blocks that were shorter (due to interruption of data acquisition) were not considered for sleep state determination. Epochs that showed a mixed sleep state (REM and quiet sleep) (which amounted to one to two epochs per study) or epochs that were staged as indeterminate sleep (15-17) by neurophysiologic and behavioral criteria were also not considered for sleep state determination using $v($ Ttot). There are two reasons for not considering indeterminate sleep: 1) because infants usually "squirm" during these periods, signals are too noisy to be useful and 2) these episodes are short and infrequent in our studies, so little information is lost by excluding indeterminate sleep.

Figure 4 gives the time course of $v$ (Ttot) during such a study in one infant. It also shows diagrammatically the sleep state $(Q$ $=$ quiet, $\mathrm{R}=\mathrm{REM}$ ) as judged by neurophysiologic signals and behavioral coding. It can be seen that during quiet sleep the

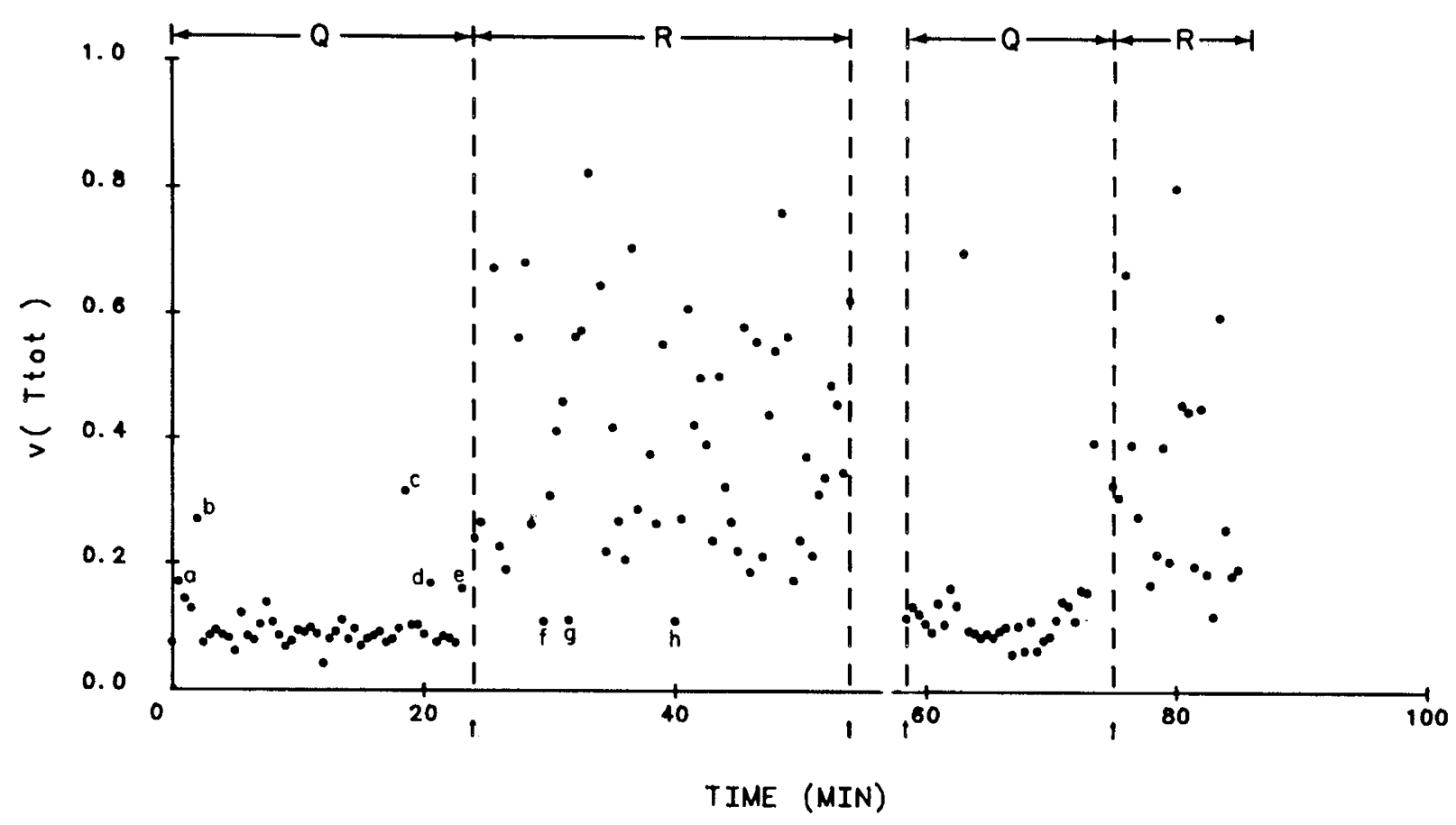

Fig. 4. Time course of v(Ttot) in one study in one infant. Dashed vertical lines (or small arrows on the x-axis) delineate the quiet (Q) or REM (R) sleep periods obtained by neurophysiologic and behavioral criteria. Note the tight distribution of $\mathrm{v}(\mathrm{Ttot})$ during quiet sleep versus the widespread distribution of $\mathrm{v}(\mathrm{Ttot})$ in REM sleep. Data points $a, b, c, d$, and $e$ are above the threshold of 0.15 chosen for the separation from REM sleep based on the Kolmogorov-Smirnov cuts for all infants studied. Data points $f, g$, and $h$ are below the same threshold (see text). 


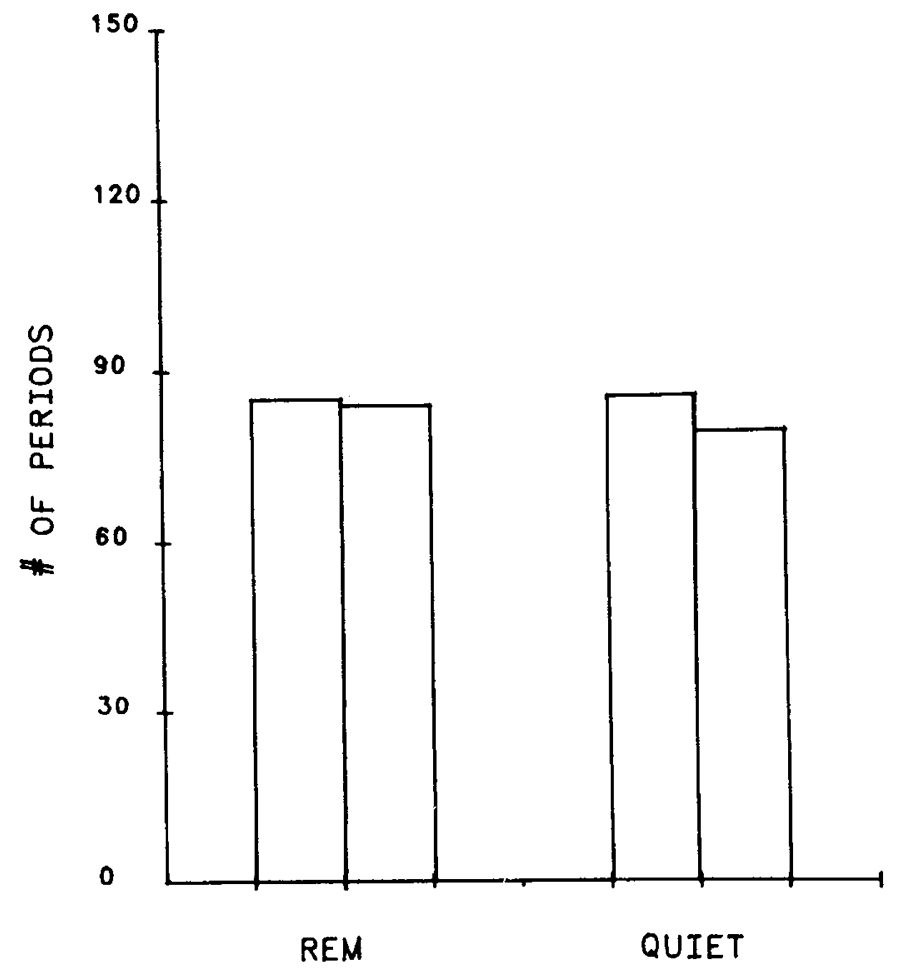

Fig. 5. Bar graphs representing the number of periods analyzed in REM sleep (first pair) and quiet sleep (second pair). The first cell of each pair represents the number of periods of REM or quiet sleep staged by neurophysiologic and behavioral criteria; the second cell represents the number of periods staged as REM or quiet sleep using the statistical decision rule we developed (see text).

overwhelming majority of data points fall below 0.15 ; only five points (a through e) are above this threshold in the first quiet cycle (Fig. 4). Note that this threshold is close to the KolmogorovSmirnov cut for this infant (infant 2) at 1 month of age which occurs at 0.16 and is similar to those of other infants (Table 2). In contrast, most data points occur above this threshold in REM sleep; only three points (f, g, h) (Fig. 4) are less than 0.15 . Moreover, these data points (i.e. those above threshold in quiet sleep and below threshold in REM sleep) are not close to each other in time. Thus, in every set of 10 consecutive time points or 5-min epochs, there are three or fewer points above the threshold in quiet sleep. Our previous analysis and some unpublished observations from our laboratory have shown that such high values of $v(T$ tot $)$ in quiet sleep are due to the perturbation in the respiratory rhythm caused by respiratory pauses present in the 5-min epochs.

In view of these results, we propose the following statistical decision rule for sleeping infants at 1 and 4 months of age.

All epoch of 5 min consisting of 10 data points [where each data point represents $v(T$ tot $)$ in a 30 -s block] is classified as quiet sleep if three or fewer data points are more than 0.15 ; otherwise the epoch is classified as REM sleep.

Performance of decision rule. There were 170 five-min epochs in the 18 studies performed on the nine normal infants at both ages. In each study, there were three to 14 epochs that were considered for determination of sleep state. Of the 85 epochs staged as quiet sleep by neurophysiologic and behavioral means, 79 epochs (or $93 \%$ ) were also classified as quiet sleep using the decision rule (Fig. 5). Of the 85 epochs staged as REM sleep by neurophysiologic and behavioral techniques, 84 were classified as REM sleep and only one was classified as quiet sleep (Fig. 5).

For the five additional infants (numbers 10-14 in Table 3) whom we used as a subsequent example to test our decision rule, of the 22 epochs staged as quiet sleep and the 21 epochs staged as REM sleep by neurophysiologic and behavioral techniques, only one was misclassified in each sleep state by our decision rule.

\section{DISCUSSION}

Automatic staging of sleep has been previously attempted using a variety of techniques and algorithms (20-22, 31-38). These previous methods most commonly based their algorithm on changes in EEG that occur in REM and quiet sleep $(34-36,38)$ or on those of the RR interval $(20,21,33,37)$. In addition, Prechtl and Lenard (39) based their sleep state analysis on the presence or absence of eye movements. In general, these methods suffer from either 1) a lack of high performance comparable to that obtained from visual sleep staging using EEG, EOG, and postural muscle EMG $(20,21,37)$ or 2 ) a lack of simplicity; some methods can be time-consuming and require the knowledge of moderately sophisticated techniques such as those employed in time series analysis of the $\operatorname{EEG}(34,35)$.

In this current work, our aim has been to develop a method that would be suitable for separating quiet from nonquiet sleep. Although it has been recognized for more than two decades that the pattern of breathing in REM sleep is different from that in quiet sleep in adult humans, infants, and animals, our analysis shows for the first time that the variability of Ttot (and not of $\mathrm{Vt}$ ) can be used to stage sleep in young infants and that this can be done with a high degree of accuracy when staging is compared with the more conventional neurophysiologic and behavioral techniques. In addition, our method has three major advantages: 1) it is simple and does not require sophisticated analysis. The only computations required are those of the mean and the SD of the population of Ttot under consideration. 2) It is based on the recording of an easily available signal in the laboratory or in a clinical setting. This signal refers to respiratory timing and not to Vt. Although our data were acquired with barometric plethysmography in order to determine whether Vt or respiratory timing is a more informative parameter, such relatively sophisticated methodologies are not needed since only respiratory timing was shown to be useful by this current analysis. There are several methods and instruments that are appropriate for recording ventilatory timing. 3 ) The threshold used to distinguish REM from quiet sleep does not seem to be sensitive to age in early life. This is evidenced by the narrow range of the KolmogorovSmirnov cuts for v(Ttot) at both ages. Although this threshold can be easily changed to adapt to the situation, the same threshold served the 1-month as well as the 4-month-old infants. As the results of Table 3 indicate, the threshold used was also adequate for the five additional infants whose data did not contribute to the formulation of the statistical decision rule. Whether older children or adults require different threshold values remains to be determined.

In this report we have shown that this method is useful when quiet sleep has to be distinguished from REM sleep. As is evident from our analysis, we focussed on epochs in which infants were asleep. Since we did not consider transitional epochs including those staged as indeterminate ones, we limited our choices to one of two states, i.e. to either REM or quiet sleep. We have not examined how this method performs when wakefulness has to be distinguished from sleep periods in infants. Since breathing in awake infants is very variable at this age, we believe that it will be difficult to separate wakefulness from REM sleep on the basis of $v$ (Ttot), even with the help of EOG. Therefore, this method separates quiet from REM sleep very well when it is known that the infant is asleep. If this information is not available, i.e. if it is unknown whether the infant is asleep or awake, this method is very useful for the extraction of quiet sleep periods from $24-\mathrm{h}$ recordings.

In summary, we have shown that it is possible to stage sleep with a high degree of accuracy in sleeping young infants using respiratory variability. The computations and the algorithm are 
simple and the instrumentation required for measuring respiratory timing is available in laboratory and clinical environments. We believe that the importance of this work stems from the fact that it is possible to stage sleep using nonneurophysiologic or behavioral criteria. It should be kept in mind, however, that we have done this analysis on normal infants at 1 and 4 months of age. Extension of these results to other populations of infants who have abnormal respiratory rhythms may be misleading. This methodology could, however, be conceivably tried on other populations and applied only after adequate investigations.

\section{REFERENCES}

1. Baust W, Bohnert B 1969 The regulation of heart rate during sleep. Exp Brain Res 7:169-180

2. Bolton DPG, Herman S 1974 Ventilation and sleep state in the newborn. J Physiol (Lond) 240:67-77

3. Curzi-Dascalova L, Gaudebout C, Dreyfus-Brisac C 1981 Respiratory frequencies of sleeping infants during the first months of life: correlations between values in different sleep states. Early Hum Dev 5:39-54

4. Finer NN, Abroms IF, Taeusch HW 1976 Ventilation and sleep states in newborn infants. J Pediatr 89:100-108

5. Haddad GG, Epstein RA, Epstein MAF, Leistner HL, Mellins RB 1980 The $R-R$ interval and $R-R$ variability in normal infants during sleep. Pediatr Res 14:809-811

6. Haddad GG, Jeng HJ, Lee SH, Lai TL 1984 Rhythmic variations in R-R interval during sleep and wakefulness in puppies and dogs. Am J Physio 247:H67-H73

7. Haddad GG, Bazzy AR, Chang SL, Mellins RB 1984 Heart rate pattern during respiratory pauses in normal infants during sleep. J Dev Physiol 6:329-337

8. Harper RM, Hoppenbrouwers T, Sterman MB, McGinty DJ, Hodgman J 1976 Polygraphic studies of normal infants during the first six months of life. I. Heart rate and variability as a function of state. Pediatr Res 10:945-95

9. Harper RM, Walter DO, Leake B, Hoffman HJ, Sieck GC, Sterman MB Hoppenbrouwers T, Hodgman J 1978 Development of sinus arrhythmia during sleeping and waking states in normal infants. Sleep 1:33-48

10. Harper RM, Leake B, Hoppenbrouwers T, Sterman MB, McGinty DJ, Hodgman J 1978 Polygraphic studies of normal infants and infants at risk for the Sudden Infant Death Syndrome. Heart rate and heart rate variability as a function of state. Pediatr Res 12:778-782

11. Hon EH, Zannini D, Quilligan EJ 1975 The neonatal value of fetal monitoring. Am J Obstet Gynecol 122:508-519

12. Leistner HL, Haddad GG, Epstein RA, Lai TL, Epstein MAF, Mellins RB 1980 Heart rate and heart rate variability during sleep in aborted sudden infant death syndrome. J Pediatr 97:51-55

13. Paul RH, Miller FC 1978 Antepartum fetal heart rate monitoring. Clin Obstet Gynecol 21:375-384

14. Rudolph AJ, Vallbona C, Desmond MM 1965 Cardiodynamic studies in the newborn. 3. Heart rate patterns in infants with idiopathic respiratory distress syndrome. Pediatrics 36:551-559

15. Anders T, Emde R, Parmelee A 1971 A Manual of Standardized Terminology, Techniques and Criteria for Scoring of States of Sleep and Wakefulness in Newborm Infants. CA:BRI Publications, Los Angeles

16. Dittrichova J 1966 The development of sleep in infancy. J Appl Physiol 21:1243-1246
17. Haddad GG, Walsh EM, Leistner HL, Grodin WK, Mellins RB 1981 Abnormal maturation of sleep states in infants with aborted Sudden Infant Death Syndrome. Pediatr Res 15:1055-1057

18. Southall DP, Richards JM, Shinebourne EA 1983 Prospective populationbased studies into heart rate and breathing patterns in newborn infants: prediction of infants at risk for SIDS? In: Tilden JT, Roeder LM, Steinschneider A (eds) Sudden Infant Death Syndrome. Academic Press, New York, pp 621-652

19. Gopal IS, Haddad GG 1981 Automatic detection of eye movements in REM sleep using the electrooculogram. Am J Physiol 241:R217-221

20. Lisenby MJ, Richardson PC, Weich AJ 1976 Detection of cyclic sleep phenomena using instantaneous heart rate. Electroencephalogr Clin Neurophysiol 40:169-177

21. Welch AJ, Richardson PC 1973 Computer sleep stage classification using heart rate data. Electroencephalogr Clin Neurophysiol 34:145-152

22. Welch AJ 1971 Period analysis of space flight EEG. Aerospace Med 42:601606

23. Harper RM, Frostig Z, Hoppenbrouwers T, Frysinger RC 1985 Relative contributions of respiratory and cardiac rate and variability measures to machine determination of sleep state in infants. Neuroscience 11:1283(abstr)

24. Harper RM, Frostig Z, Hoppenbrouwers T, Frysinger RC 1985 Machine classification of infant sleep states utilizing cardiorespiratory measures. Sleep Res 14:73(abstr)

25. Haddad GG, Epstein RA, Epstein MAF, Leistner HL, Marino PA, Mellins RB 1979 Maturation of ventilation and ventilatory pattern in normal sleeping infants. J Appl Physiol 46:998-1002

26. Law HS, Epstein RA, Epstein MAF 1979 High resolution determination of the RR interval. Am J Physiol 236:894-898

27. Epstein RA, Epstein MAF, Haddad GG, Mellins RB 1980 Practical implementation of the barometric method for measurement of tidal volume. J Appl Physiol 49:1107-1115

28. Sokal RR, Rohlf FJ 1969 Biometry, 2nd ed. WH Freeman and Co, Philadelphia

29. Gordon L, Olshen RA 1978 Asymptotically efficient solutions to the classification problem. Ann Statist 6:515-533

30. Stoller DS 1954 Univariate two-population distribution-free discrimination. J Am Statist Assoc 49:770-777

31. Frost Jr JD 1970 An automatic sleep analyzer. Electroencephalogr Clin Neurophysiol 29:88-92

32. Gaillard JM, Tissot R 1973 Principles of automatic analysis of sleep records with a hybrid system. Comput Biomed Res 6:1-13

33. Goodlin RC, Schmidt W 1972 Human fetal arousal levels as indicated by heart rate recordings. Am J Obstet Gynecol 5:613-621

34. Itil TM, Shapiro DM, Fink M, Kassebaum M 1969 Digital computer classifications of EEG sleep stages. Electroencephalogr Clin Neurophysiol 27:7683

35. Larsen LE, Walter DO 1970 On automatic methods of sleep staging by EEG spectra. Electroencephalogr Clin Neurophysiol 28:459-467

36. Lubin A, Johnson LC, Austin MT 1969 Discrimination among states of consciousness using EEG spectra. Psychophysiology 6:122-131

37. Mann LI, Duchin S, Weiss RR 1974 Fetal EEG sleep stages and physiologic variability. Am J Obstet Gynecol 4:533-538

38. Martin WB, Johnson LC, Viglione SS, Naitoh P, Joseph RD, Moses JD 1972 Pattern recognition of EEG-EOG as a technique for all-night sleep stage scoring. Electroencephalogr Clin Neurophysiol 32:417-427

39. Prechtl HFR, Lenard HG 1967 A study of eye movements in sleeping newborn infants. Brain Res 5:477-493

40. Roessler R, Collins F, Ostman R 1970 A period analysis classification of sleep stages. Electroencephalogr Clin Neurophysiol 29:358-362 